\title{
Non-expander Cayley graphs of simple groups
}

\author{
Gábor Somlai \\ Alfréd Rényi Institute of Mathematics \\ email: zsomlei@gmail.com*
}

\begin{abstract}
For every infinite sequence of simple groups of Lie type of growing rank we exhibit connected Cayley graphs of degree at most 10 such that the isoperimetric number of these graphs converges to 0 . This proves that these graphs do not form a family of expanders.
\end{abstract}

\section{Introduction}

Let $G$ be a finite group and $T$ a subset of $G$. The Cayley graph $\operatorname{Cay}(G, T)$ is defined by having vertex set $G$ and $g$ is adjacent to $h$ if and only if $g^{-1} h \in T$. A Cayley graph $\operatorname{Cay}(G, T)$ is undirected if and only if $T=T^{-1}$, where $T^{-1}=$ $\left\{t^{-1} \in G \mid t \in T\right\}$.

Let $\Gamma$ be an arbitrary graph and $S \subseteq V(\Gamma)$. We define the boundary of $S$ which we denote by $\partial S$ to be the set of vertices in $V(\Gamma) \backslash S$ with at least one neighbour in $S$. For a graph $\Gamma$ the isoperimetric number $h(\Gamma)$ is defined by

$$
h(\Gamma)=\min \left\{\frac{|\partial S|}{|S|}|S \subset V(\Gamma), 0<| S \mid \leq \frac{|V(\Gamma)|}{2}\right\} .
$$

A graph $\Gamma$ is called an $\epsilon$-expander if $h(\Gamma) \geq \epsilon$ and a series of $k$-regular graphs $\Gamma_{n}$ is called an expander family if there is a constant $\epsilon>0$ such that for every $n$ the graph $\Gamma_{n}$ is an $\epsilon$-expander. Finally, we say that a family of groups $G_{n}$ is a family of uniformly expanding groups if there exist $0<\epsilon \in \mathbb{R}$ and $k \in \mathbb{N}$ such that for every $i$ and every generating set $S_{i} \subset G_{i}$ of size at most $k$ the Cayley graphs $\operatorname{Cay}\left(G_{i}, S_{i}\right)$ are $\epsilon$-expanders.

The study of series of Cayley graphs of finite simple groups has received great attention. The proof of the fact was completed by Kassabov, Lubotzky and Nikolov in [3] based on several earlier work (see [4, $[5,,[6],[8,[12]$ ), that there exist $k \in \mathbb{N}$ and $0<\epsilon \in \mathbb{R}$ such that every non-abelian finite simple group which is not a Suzuki group has a set of generators $S$ of size at most $k$ for which $\operatorname{Cay}(G, S)$ is an $\epsilon$-expander. This work was extended by Breuillard, Green and

*Research is partially supported by MTA Renyi "Lendulet" Groups and Graphs Research Group 
Tao in [1] to the Suzuki groups. These results can also motivate the question which was asked by Lubotzky in [11] whether every family of Chevalley groups of bounded rank is a family of uniformly expanding group.

Lubotzky also suggested to investigate families of simple groups of unbounded rank in [10] and wrote that it seems likely that if $G_{n}$ is a sequence of non-abelian simple groups such that the rank of $G_{n}$ is unbounded, then for every $n$ there exists a generating set $T_{n} \subset G_{n}$ such that the graphs $\operatorname{Cay}\left(G_{n}, T_{n}\right)$ do not form a family of expanders. An explicit example (see [9]) of a non-expander family of Cayley graphs of special linear groups was given by Luz. The diameter of the graphs given by Luz was investigated by Kassabov an Riley and it was proved in [7] that there exists $c \in \mathbb{R}$ such that the diameter of the graphs is smaller than $c \log (|S L(n, p)|)$. Similarly, the symmetric group $S_{n}$ is generated by $\gamma=(12)$ and $\sigma_{n}=(1,2, \ldots, n)$ for every $n \in \mathbb{N}$ and the sequence of isoperimetric numbers $h\left(\operatorname{Cay}\left(S_{n}, \gamma, \sigma_{n}\right)\right)$ tends to 0 , see [9]. Moreover, one can find a set of generators of $S_{n}$ such that the diameter of the corresponding Cayley graphs is $\Omega\left(n^{2}\right)$ which gives that these Cayley graphs do not form a family of expanders.

We will investigate 7 series $\left(A_{l}, B_{l}, C_{l}, D_{l}, A_{2 n-1}^{1}, A_{2 n}^{1}, D_{n}^{1}\right)$ of finite simple groups of Lie type. These are the groups of Lie type such that the rank of a sequence of groups tends to infinity if we fix a series of the Lie group. In order to define generators and subgroups of these groups we will use the generators given by Steinberg in [13 and we will use the notation and several results of the book of Carter [2].

For these 7 series of finite simple groups of Lie type we construct Cayley graphs and subsets such that the number of the neighbours of these subsets depends on the rank of the groups. Moreover, the isoperimetric number of these graphs tends to 0 . This proves the conjecture of Lubotzky concerning the series of Cayley graphs of simple groups of unbounded rank. More precisely, we prove the following:

Theorem 1. (a) Let $G$ be a Chevalley group of rank $l$ of type $A_{l}, B_{l}, C_{l}$ or $D_{l}$. For every $l \geq 5$ and for every finite field $G F(q)$ there exists a generating set $T$ of cardinality at most 10 and a subset of the vertices $S \subset V(C a y(G, T))$ with $|S| \leq \frac{|G|}{2}$ such that $\frac{|\partial(S)|}{|S|} \leq \frac{6}{l-3}$.

(b) Let $G$ be a twisted group of type $A_{2 n-1}^{1}, D_{n}^{1}$ or $A_{2 n}^{1}$. For every $n \geq 5$ and for every finite field $G F(q)$ there exists a generating set $T^{\prime}$ of cardinality at most 8 and $S^{\prime} \subset V\left(\operatorname{Cay}\left(G, T^{\prime}\right)\right)$ with $\left|S^{\prime}\right| \leq \frac{|G|}{2}$ such that $\frac{\left|\partial\left(S^{\prime}\right)\right|}{\left|S^{\prime}\right|} \leq \frac{6}{n-2}$.

The paper is organized into the following 4 sections. In Section 2 we give all necessary definitions and we collect some important fact about the construction of simple groups of Lie type. The proof of Theorem 1 (a) is contained in Section 3 and Theorem 1 (b) which is the case of twisted groups will be handled in Section 4. In Section 5 we present the original construction in terms of matrices which was extended to several different series of simple groups. 


\section{Preliminaries}

In this section we collect important facts about about finite simple groups of Lie-type and we build up the notation we will use all along this paper.

Let $K=G F(q)$ be a finite field. We denote by $\Phi$ the system of roots and $\Phi=\Phi^{+} \cup \Phi^{-}$is the union of the positive and negative roots. We also choose $\Pi=\left\{r_{1}, r_{2}, \ldots, r_{l}\right\} \subset \Phi^{+}$which is the set of the fundamental roots.

The Weyl group $W$ is generated by the fundamental reflections $w_{r}$, where $r \in \Pi$. In order to simplify notation we denote by $w_{i}$ the fundamental reflections $w_{r_{i}}$, where $r_{i} \in \Pi$. We denote by $x_{r}(\psi)$ the standard generators of the Chevalley group $G$, where $r \in \Phi$ and $\psi \in K$. If $r=r_{i}$ for some $r_{i} \in \Pi$, then we denote by $x_{i}(\psi)$ the standard generator $x_{r_{i}}(\psi)$. These elements generate the Chevalley group $G$. The subgroups $X_{r}=\left\{x_{r}(t) \mid t \in K\right\}$ are called root subgroups of $G$ if $r \in \Phi$.

The Weyl group $W$ is isomorphic to $N / H$ for some $H \triangleleft N \leq G$. The cosets of $H$ in $N$ can be written as $n_{w} H$ for all $w \in W$ and $N$ is generated by $H$ and the elements $n_{r}$ for $r \in \Phi$. Moreover, $n_{r}=x_{r}(1) x_{-r}(-1) x_{r}(1)$ is the element of the subgroup generated by the root subgroups $X_{r}$ and $X_{-r}$. It is well known that $n_{r} x_{s}(t) n_{r}^{-1}=x_{w_{r}(s)}\left(\eta_{r, s} t\right)$ for some $\eta_{r, s} \in K$ depending only on $r$ and $s$, see [2, p.101.]. The elements of the normal subgroup $H$ of $N$ can be written in the form $h(\chi)$ where $\chi$ is a $K$-character of $\mathbb{Z} \Phi$. The subgroup $H$ is generated by the elements of the set $\left\{h_{r}(\lambda) \mid r \in \Phi\right.$ and $\left.\lambda \in K^{*}\right\}$, where the $K$-character corresponding to $h_{r}(\lambda)$ is $\chi_{r, \lambda}$ with $\chi_{r, \lambda}(a)=\lambda^{\frac{2(a, r)}{(r, r)}} . H$ is a normal subgroup of $N$ and $n_{w} h(\chi) n_{w}^{-1}=h\left(\chi^{\prime}\right)$, where $\chi^{\prime}(r)=\chi\left(w^{-1}(r)\right)$ see 2. p.102.]. Furthermore, $h_{r}(\lambda)=n_{r}(\lambda) n_{r}(-1)$ and hence $h_{r}(\lambda) \in\left\langle X_{r}, X_{-r}\right\rangle$, see [2, p.96.].

\section{Chevalley groups}

In this section we construct series of Cayley graphs for 4 different series of Chevalley groups. For these Chevalley groups we need 6 series of Cayley graphs. The six different constructions are similar but we will treat them separately.

We first prove the following technical lemma.

Lemma 1. Let $w=w_{1} w_{2} \ldots w_{l}$ be a Coxeter element of the Weyl group $W$ and let us assume that the fundamental root $r_{i}$ is orthogonal to $r_{j}$ if $i+1<j \leq l$ and $r_{i+1}$ is orthogonal to $r_{k}$ if $1 \leq k \leq i-1$. We also assume that $r_{i}$ and $r_{i+1}$ have the same length and $w_{i}\left(r_{i+1}\right)=r_{i}+r_{i+1}$. Then $w\left(r_{i}\right)=r_{i+1}$.

Proof. Since $r_{i}$ is orthogonal to $r_{j}$ for every $j>i+1$ we have that $w\left(r_{i}\right)=$ $w_{1} w_{2} \ldots w_{i} w_{i+1}\left(r_{i}\right)$. The elements $w_{k}$ are reflections through the hyperplane perpendicular to $r_{k}$. Thus $w_{k}\left(r_{k}\right)=-r_{k}$ for every $1 \leq k \leq l$ and $w_{i+1}\left(r_{i}\right)=$ $r_{i}+r_{i+1}=w_{i}\left(r_{i+1}\right)$ since $r_{i}$ and $r_{i+1}$ have the same length. It follows that $w_{i} w_{i+1}\left(r_{i}\right)=w_{i}\left(r_{i}+r_{i+1}\right)=w_{i}\left(r_{i}\right)+w_{r_{i+1}}=-r_{i}+\left(r_{i}+r_{i+1}\right)=r_{i+1}$. Hence $w\left(r_{i}\right)=w_{1} w_{2} \ldots w_{i-1}\left(r_{i+1}\right)=r_{i+1}$ since $r_{i+1}$ is orthogonal to $r_{k}$ for $1 \leq k \leq$ $i-1$. 


\section{$3.1 A_{l}$}

Let $G$ be a Chevalley group of type $A_{l}$. The Dynkin diagram of the corresponding root system is the following.

$\stackrel{r_{1}}{\circ} \quad \stackrel{r_{2}}{\circ} \quad \stackrel{r_{3}}{\circ}-\cdots-\stackrel{r_{l-1}}{\circ} \stackrel{r_{l}}{\circ}$

One can see from the Dynkin diagram that $w_{i}\left(r_{i+1}\right)=r_{i}+r_{i+1}=w_{i+1}\left(r_{i}\right)$ for $i=1, \ldots, l-1$.

Let $w=w_{1} w_{2} \ldots w_{l}$ be a Coxeter element of the Weyl group. We choose $\lambda$ to be a generator of the multiplicative group of $G F(q)$.

Lemma 2. $x_{1}(1), n_{w}$ and $h_{r_{1}}(\lambda)$ generate the Chevalley group $G$.

Proof. It was proved in [13] that $x_{1}(1) n_{w}$ and $h_{r_{1}}(\lambda)$ generate $G$. Clearly, $x_{1}(1)$ and $n_{w}$ generate $x_{1}(1) n_{w}$ which proves the Lemma.

For every $l \geq 5$ we define the following undirected Cayley graph:

$$
\Gamma_{a}=\operatorname{Cay}\left(G,\left\{x_{1}(1), n_{w}, h_{r_{1}}(\lambda), x_{1}(1)^{-1}, n_{w}^{-1}, h_{r_{1}}(\lambda)^{-1}\right\}\right) .
$$

Let $K_{a}$ be the subgroup of the Chevalley group $G$ generated by the root subgroups $X_{r_{1}}, X_{-r_{1}}, X_{2}, X_{-r_{2}}, \ldots, X_{r_{l-1}}, X_{-r_{l-1}}$ and let

$$
S_{a}=\cup_{i=0}^{l-1} K_{a} n_{w}^{i} .
$$

Every element of the Weyl group $W$ acts on the the root system $\Phi$.

Lemma 3. The orbit of $w$ which contains $r_{1}$ is the following:

$$
\rightarrow r_{1} \stackrel{w}{\longrightarrow} r_{2} \stackrel{w}{\longrightarrow} r_{3} \stackrel{w}{\longrightarrow} \cdots \stackrel{w}{\longrightarrow} r_{l-1} \stackrel{w}{\longrightarrow} r_{l} \stackrel{w}{\longrightarrow}-r_{1}-\ldots-r_{l} \stackrel{w}{\longrightarrow}
$$

This can be formulated as follows:

$$
\begin{aligned}
w\left(r_{i}\right) & =r_{i+1} \text { for } 1 \leq i \leq l-1 \\
w\left(r_{l}\right) & =-r_{1}-\ldots-r_{l} \\
w\left(-r_{1}-r_{2}-\ldots-r_{l}\right) & =r_{1}
\end{aligned}
$$

Proof. Lemma 19ives that $w\left(r_{i}\right)=r_{i+1}$ for $1 \leq i \leq n-1$ and

$$
w\left(r_{l}\right)=w_{1} w_{2} \ldots w_{l}\left(r_{l}\right)=w_{1} w_{2} \ldots w_{l-1}\left(-r_{l}\right)=-w_{1} w_{2} \ldots w_{l-1}\left(r_{l}\right)
$$

since $w$ is a linear transformation of the vector space spanned by the roots. We also have $w_{j}\left(r_{j+1}+\cdots+r_{l}\right)=r_{j}+r_{j+1}+\cdots+r_{l}$ for $1 \leq j \leq l-1$. Therefore

$$
\begin{aligned}
w_{1} w_{2} \ldots w_{l-1}\left(r_{l}\right) & =w_{1} w_{2} \ldots w_{l-2}\left(r_{l-1}+r_{l}\right) \\
& =w_{1} w_{2} \ldots w_{l-3}\left(r_{l-2}+r_{l-1}+r_{l}\right)=\ldots=r_{1}+r_{2}+\ldots+r_{l} .
\end{aligned}
$$

This shows that

$$
w\left(r_{l}\right)=-\left(r_{1}+r_{2}+\ldots+r_{l}\right) .
$$


Using again the linearity of $w$ and equation (1) we get

$$
w\left(r_{1}+r_{2}+\ldots+r_{l}\right)=r_{2}+r_{3}+\ldots+r_{l}-\left(r_{1}+r_{2}+\ldots+r_{l}\right)=-r_{1} .
$$

This gives $w\left(-\left(r_{1}+r_{2}+\ldots+r_{l}\right)\right)=r_{1}$, finishing the proof Lemma 3 ,

It follows from Lemma 3 that if $1 \leq i \leq l-1$, then $n_{w}^{i} K_{a} n_{w}^{-i}$ contains $n_{w}^{i} X_{r_{l-i}} n_{w}^{-i}=X_{r_{l}}$. Therefore $n_{w}^{i} K_{a} n_{w}^{-i} \neq K_{a}$ which shows that $n_{w}^{i} \notin K_{a}$ for every $1 \leq i \leq l-1$. This implies that $K_{a}, K_{a} n_{w}, \ldots, K_{a} n_{w}^{l-1}$ are different right cosets of $K_{a}$ so $S_{a}$ is the union of $l$ pairwise disjoint subsets of the vertices of $\Gamma_{a}$ and these subsets have the same cardinality.

Lemma 4. $\frac{\left|\partial\left(S_{a}\right)\right|}{\left|S_{a}\right|} \leq \frac{6}{l}$

Proof. $S_{a}$ is the union of $l$ right cosets of $K_{a}$ so $\left|S_{a}\right|=l\left|K_{a}\right|$. It is clear from the definition of $S_{a}$ that $\left(K_{a} n_{w}^{i}\right) n_{w} \subset S_{a}$ for every $0 \leq i \leq l-2$ and similarly $\left(K_{a} n_{w}^{i}\right) n_{w}^{-1} \subset S_{a}$ if $1 \leq i \leq l-1$. Therefore those neighbors of $S_{a}$ which are not in $S_{a}$ can only be obtained as an element of following subset of the vertices of $\Gamma_{a}$ :

$$
\begin{aligned}
& K_{a} n_{w}^{l} \bigcup K_{a} n_{w}^{-1} \bigcup_{i=1}^{l-1}\left(K_{a} n_{w}^{i}\right) x_{1}(1) \bigcup_{i=1}^{l-1}\left(K_{a} n_{w}^{i}\right) x_{1}(1)^{-1} \bigcup_{i=1}^{l-1}\left(K_{a} n_{w}^{i}\right) h_{r}(\lambda) \\
& \bigcup_{i=1}^{l-1}\left(K_{a} w^{i}\right) h_{r}(\lambda)^{-1} .
\end{aligned}
$$

$K_{a}$ is a subgroup of $G$ so $\left(K_{a} n_{w}^{i}\right) x=K_{a} n_{w}^{i}$ if and only if $n_{w}^{i} x n_{w}^{-i} \in K_{a}$. We first apply this observation to $x_{1}(1)$ and $x_{1}(1)^{-1}=x_{1}(-1)$. It is easy to see from Lemma 3 that $n_{w}^{i} x_{1}( \pm 1) n_{w}^{-i}$ is of the form $x_{w^{i}\left(r_{1}\right)}(\alpha)=x_{i+1}(\alpha)$ for some $\alpha \in G F(q)^{*}$ if $0 \leq i \leq l-1$. It follows that $n_{w}^{i} x_{1}( \pm 1) n_{w}^{-i} \in X_{r_{i+1}} \subset K_{a}$ if $i \neq l-1$.

Using the fact that $h_{r}(\lambda)$ and $h_{r}(\lambda)^{-1}$ are in the subgroup $\left\langle X_{r}, X_{-r}\right\rangle$ we get that $n_{w}^{i} h_{r_{1}}(\lambda)^{ \pm 1} n_{w}^{-i} \in\left\langle X_{w^{i}\left(r_{1}\right)}, X_{-w^{i}\left(r_{1}\right)}\right\rangle=\left\langle X_{r_{i+1}}, X_{-r_{i+1}}\right\rangle \subset K_{a}$ if $i \neq l-1$.

Now, $\partial S_{a} \subseteq K_{a} n_{w}^{l} \cup K_{a} n_{w}^{-1} \cup K_{a} n_{w}^{l-1} x_{1}(1) \cup K_{a} n_{w}^{l-1} x_{1}(-1) \cup K_{a} n_{w}^{l-1} h_{r_{1}}(\lambda) \cup$ $K_{a} n_{w}^{l-1} h_{r_{1}}\left(\frac{1}{\lambda}\right)$. These subsets are all of them right cosets of $K_{a}$ so they have the same cardinality which proves that $\left|\partial S_{a}\right| \leq 6\left|K_{a}\right|$, while $\left|S_{a}\right|=l\left|K_{a}\right|$.

Remark 1. In order to prove Theorem $1(a)$ we repeat the previous construction several times. In every single case the connection set of the Cayley graph will consist of few standard generators of the Chevalley group, an element of the form $n_{w}$, where $w=w_{1} w_{2} \ldots w_{l}$ is a Coxeter element of the corresponding Weyl group and an element of the group $H$. If $G$ is of rank $l$ we will choose a subgroup of $G$ which is isomorphic to a Chevalley group of rank $l-1$ and which is of the same type. The subset of the vertices for which the isoperimetric number is sufficiently small will be the union of cosets of the subgroup of rank $l-1$. 


\section{$3.2 \quad B_{l}$}

Let $G$ be a Chevalley group of type $B_{l}$ The Dynkin diagram of a Chevalley group of type $B_{l}$ is the following:

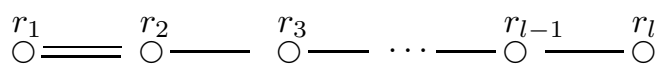

It is easy to see from the Dynkin diagram that $w_{1}\left(r_{2}\right)=r_{2}+2 r_{1}$ and $w_{2}\left(r_{1}\right)=$ $r_{1}+r_{2}$.

One can see using Lemma 1 that

$$
w\left(r_{i}\right)=w_{1} w_{2} \ldots w_{l}\left(r_{i}\right)=r_{i+1} \text { for } 2 \leq i \leq l-1 .
$$

The fundamental roots $r_{3}, \ldots, r_{l}$ are orthogonal to $r_{1}$. Therefore $w\left(r_{1}\right)=$ $w_{1} w_{2}\left(r_{1}\right)=w_{1}\left(r_{1}+r_{2}\right)=-r_{1}+\left(r_{2}+2 r_{1}\right)=r_{1}+r_{2}$. We also have that $w$ is linear so using equation (2) we have that if $2 \leq j \leq l-1$, then

$$
w\left(r_{1}+r_{2}+\ldots+r_{j}\right)=w\left(r_{1}\right)+w\left(r_{2}\right)+\ldots+w\left(r_{j}\right)=r_{1}+r_{2}+r_{3}+\ldots+r_{j+1} .
$$

Using these observations we conclude that the following picture represents a part of the orbit of the action of the group generated by $w$ including the root $r_{1}$ :

$$
r_{1} \stackrel{\mathrm{w}}{\longrightarrow} r_{1}+r_{2} \stackrel{\mathrm{w}}{\longrightarrow} r_{1}+r_{2}+r_{3} \stackrel{\mathrm{w}}{\longrightarrow} \cdots \longrightarrow r_{1}+r_{2}+\ldots+r_{l} \stackrel{\mathrm{w}}{\longrightarrow}
$$

This can be formulated as follows:

$$
w^{i}\left(r_{1}\right)=r_{1}+r_{2}+\ldots+r_{i+1} \text { for } i=1, \ldots, l-1 .
$$

The orbit of $\langle w\rangle$ containing these elements contains $w\left(r_{1}+r_{2}+\ldots+r_{l}\right)$ as well. It is easy to see that $w_{i}\left(r_{i+1}+r_{i+2}+\ldots+r_{l}\right)=r_{i}+r_{i+1}+\ldots+r_{l}$ if $2 \leq i \leq l-1$. We also have $w_{1}\left(r_{2}\right)=2 r_{1}+r_{2}$ hence

$$
\begin{aligned}
w\left(r_{l}\right) & =w_{1} \ldots w_{l-1} w_{l}\left(r_{l}\right)=-w_{1} \ldots w_{l-1}\left(r_{l}\right) \\
& =-w_{1} \ldots w_{l-2}\left(r_{l-1}+r_{l}\right)=\ldots=-w_{1}\left(r_{2}+\ldots+r_{l}\right) \\
& =-\left(r_{l}+\ldots+r_{2}+2 r_{1}\right) .
\end{aligned}
$$

This implies using equation (3) that

$$
w\left(r_{1}+\ldots+r_{l}\right)=w\left(r_{1}+\ldots+r_{l-1}\right)+w\left(r_{l}\right)=-r_{1} .
$$

One can easily describe the remaining elements of the orbit since $w$ is linear.

We also investigate the action of $\langle w\rangle$ on $2 r_{1}+r_{2}+\ldots+r_{l}$ and $r_{2}+\ldots+r_{l}$. Using equation (5) and the linearity of $w$ we get that $w\left(2 r_{1}+r_{2}+\ldots+r_{l}\right)=$ $w\left(r_{1}\right)+w\left(r_{1}+r_{2}+\ldots+r_{l}\right)=r_{1}+r_{2}-r_{1}=r_{2}$. It follows using equation (2) that

$$
w^{i}\left(2 r_{1}+r_{2}+\ldots+r_{l}\right)=r_{i+1} \text { for } 1 \leq i \leq l-1 .
$$

One can also prove using equation (4) and equation (6) that

$$
w^{i}\left(r_{2}+\ldots+r_{l}\right)=-2\left(r_{1}+r_{2}+\ldots+r_{i+1}\right)+r_{i+1} \text { for } 1 \leq i \leq l-1 .
$$




\subsection{1 $\operatorname{Char}(K)>2$}

Let us assume that $\operatorname{char}(K)>2$.

Lemma 5. $x_{1}(1), n_{w}$ and $h_{t}(\lambda)$, where $t=2 r_{1}+r_{2}+\cdots+r_{l}$ generate the Chevalley group $G$ of type $B_{l}$ if the characteristic of the underlying field is not 2.

Proof. It was proved in 13 that $x_{1}(1) n_{w}$ and $h_{t}(\lambda)$ generate the Chevalley group $G$ if $\operatorname{char}(K) \neq 2$.

We define again a sequence of connected Cayley graphs. Let

$$
\Gamma_{b}=\operatorname{Cay}\left(G,\left\{x_{1}(1), x_{1}(-1), n_{w}, n_{w}^{-1}, h_{t}(\lambda), h_{t}(\lambda)^{-1}\right\}\right),
$$

where $G$ is of rank $l$ and $w=w_{1} w_{2} \ldots w_{l}$. Similarly to the previous case let

$$
K_{b}=\left\langle X_{r_{1}}, X_{-r_{1}}, X_{r_{2}}, X_{-r_{2}}, \ldots, X_{r_{l-1}}, X_{-r_{l-1}}\right\rangle
$$

and let

$$
S_{b}=\cup_{i=0}^{l-2} K_{b} n_{w}^{i}
$$

Lemma 6. $\frac{\left|\partial\left(S_{b}\right)\right|}{\left|S_{b}\right|} \leq \frac{4}{l-1}$

Proof. We claim that $S_{b}$ is the union of pairwise disjoint right cosets of the same subgroup $K_{b}$ in $G$. We only have to show that $n_{w}^{i} \notin K_{b}$ if $1 \leq i \leq l-2$. Straightforward calculation shows using equation (2) that $n_{w}^{i} X_{r_{l-i}} n_{w}^{-i}=X_{r_{l}}$ if $1 \leq i \leq l-2$. Therefore $X_{r_{l}} \subset n_{w}^{i} K_{b} n_{w}^{-i} \neq K_{b}$ if $1 \leq i \leq l-2$ which gives that $n_{w}^{i} \notin K_{b}$. Thus $S_{b}$ is the union of $l-1$ pairwise disjoint right cosets of $K_{b}$.

Using the definition of the Cayley graph $\Gamma_{b}$ we have that $\partial S_{b}$ is a subset of the following set:

$$
\begin{aligned}
& \bigcup_{i=0}^{l-2}\left(K_{b} n_{w}^{i}\right) n_{w} \bigcup_{i=0}^{l-2}\left(K_{b} n_{w}^{i}\right) n_{w}^{-1} \bigcup_{i=0}^{l-2}\left(K_{b} n_{w}^{i}\right) x_{1}(1) \bigcup_{i=0}^{l-2}\left(K_{b} n_{w}^{i}\right) x_{1}(-1) \\
& \bigcup_{i=0}^{l-2}\left(K_{b} n_{w}^{i}\right) h_{t}(\lambda) \bigcup_{i=0}^{l-2}\left(K_{b} n_{w}^{i}\right) h_{t}(\lambda)^{-1} .
\end{aligned}
$$

By the definition of $S_{b}$ the subsets $K_{b} n_{w}^{i} n_{w}$ are contained in $S_{b}$ if $0 \leq i \leq l-3$ and $K_{b} n_{w}^{i} n_{w}^{-1} \subset S_{b}$ if $1 \leq i \leq l-2$.

Using equation (41) we have $n_{w}^{i} x_{1}( \pm 1) n_{w}^{-i}=x_{r_{1}+r_{2}+\ldots+r_{i+1}}(t)$ for some $t \in$ $K^{*}$. If $0 \leq i \leq l-2$, then $x_{r_{1}+r_{2}+\ldots+r_{i+1}}(t) \in K_{b}$ since $r_{1}+r_{2}+\ldots+r_{i+1}$ is in the root system generated by the fundamental roots $r_{1}, r_{2}, \ldots, r_{l-1}$ and $K_{b}$ is the Chevalley group of type $B_{l-1}$ generated by the corresponding root subgroups. Therefore $K_{b} n_{w}^{i} x_{1}( \pm 1)=K_{b} n_{w}^{i} \subset S_{b}$ if $0 \leq i \leq l-2$.

The elements $h_{t}(\lambda)$ and $h_{t}(\lambda)^{-1}=h_{t}\left(\frac{1}{\lambda}\right)$ are in the subgroup generated by $X_{t}$ and $X_{-t}$. Equation (6) shows that $n_{w}^{i} X_{t} n_{w}^{-i}=X_{w^{i}(t)}=X_{r_{i+1}}$ and by the linearity of $w$ we have $n_{w}^{i} X_{-r} n_{w}^{-i}=X_{-r_{i+1}}$ for $i=1,2, \ldots, l-2$. Thus $n_{w}^{i} h_{t}(\lambda) n_{w}^{-i}$ and $n_{w}^{i} h_{t}\left(\frac{1}{\lambda}\right) n_{w}^{-i}$ are in $\left\langle X_{r_{i+1}}, X_{-r_{i+1}}\right\rangle \leq K_{b}$ if $1 \leq i \leq l-2$.

It follows that $\partial S_{b} \subset K_{b} n_{w}^{l-1} \cup K_{b} n_{w}^{-1} \cup K_{b} h_{t}(\lambda) \cup K_{b} h_{t}\left(\frac{1}{\lambda}\right)$ which gives $\frac{\left|\partial S_{b}\right|}{\left|S_{b}\right|} \leq \frac{4\left|K_{b}\right|}{(l-1)\left|K_{b}\right|}=\frac{4}{l-1}$. 


\subsection{2 $\operatorname{Char}(K)=2$}

Lemma 7. $x_{s}(1), x_{-r_{1}}(1), n_{w}$ and $h_{t}(\lambda)$, where $s=r_{2}+\cdots+r_{l}$ generate the Chevalley group $G$ of type $B_{l}$ if char $(K)=2$.

Proof. It was proved in 13 that $x_{s}(1) x_{-r_{1}}(1) n_{w}$ and $h_{t}(\lambda)$ generate $G$ if $K=$ $G F\left(2^{k}\right)$ with $k>1$ and $x_{s}(1) x_{-r_{1}}(1)$ and $n_{w}$ generate $G$ if $|K|=2$.

Let

$$
\Gamma_{b}^{\prime}=\operatorname{Cay}\left(G,\left\{x_{s}(1), x_{-r_{1}}(1), n_{w}^{ \pm 1}, h_{t}(\lambda)^{ \pm 1}\right\}\right) .
$$

The set $S_{b}$ can be considered as a subset of $V\left(\Gamma_{b}^{\prime}\right)$ so we claim the following.

Lemma 8. $\frac{\left|\partial\left(S_{b}\right)\right|}{\left|S_{b}\right|} \leq \frac{5}{l-1}$

Proof. It was proved in Lemma 6 that $\left|S_{b}\right|=(l-1)\left|K_{b}\right|$.

Similarly, the proof of Lemma 6 shows that $K_{b} n_{w}^{i} h_{t}(\lambda)^{ \pm 1} \subset S_{b}$ if $1 \leq i \leq$ $l-2$. By the definition of $S_{b}$ we have $K_{b} n_{w}^{i} n_{w} \subset S_{b}$ if $0 \leq i \leq l-3$ and $K_{b} n_{w}^{i} n_{w}^{-1} \subset S_{b}$ if $1 \leq i \leq l-2$.

Using $w\left(-r_{1}\right)=-w\left(r_{1}\right)$ and equation (4) we get that $n_{w}^{i} x_{-r_{1}}(1) n_{w}^{-i} \in K_{b}$ if $0 \leq i \leq l-2$ since $w^{i}\left(r_{1}\right)=r_{1}+r_{2}+\ldots+r_{i+1}$ by equation (4). Hence $K_{b} n_{w}^{i} x_{-r_{1}}(1)=K_{b} n_{w}^{i} \subset S_{b}$.

Equation (7) shows that $n_{w}^{i} x_{s}(1) n_{w}^{-i}$ is in $K_{b}$ if $1 \leq i \leq l-2$. Therefore $\left(\cup_{i=1}^{l-2} K_{b} n_{w}^{i}\right) x_{s}(1) \subset S_{b}$. Finally, we conclude that $\partial\left(S_{b}\right) \subset K_{b} n_{w}^{-1} \cup K_{b} n_{w}^{l-1} \cup$ $K_{b} h_{t}(\lambda) \cup K_{b} h_{t}(\lambda)^{-1} \cup K_{b} x_{s}(1)$.

\section{$3.3 C_{l}$}

The Dynkin diagram is the following in this case:

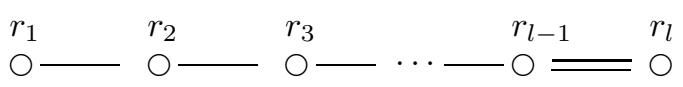

It can easily be verified using the Dynkin diagram that $w_{l-1}\left(r_{l}\right)=r_{l}+2 r_{l-1}$ and $w_{l}\left(r_{l-1}\right)=r_{l-1}+r_{l}$.

Using Lemma 1 one can see that $w\left(r_{i}\right)=r_{i+1}$ for $i=1,2, \ldots, l-2$. We also have

$$
\begin{aligned}
w\left(r_{l-1}\right) & =w_{1} w_{2} \ldots w_{l}\left(r_{l-1}\right)=w_{1} w_{2} \ldots w_{l-1}\left(r_{l}+r_{l-1}\right) \\
& =w_{1} w_{2} \ldots w_{l-2}\left(r_{l}+r_{l-1}\right) .
\end{aligned}
$$

Since $r_{l}$ is orthogonal to the remaining roots $r_{1}, r_{2}, \ldots, r_{l-2}$ we have

$$
w\left(r_{l-1}\right)=r_{l}+w_{1} w_{2} \ldots w_{l-2}\left(r_{l-1}\right) .
$$

Since $w_{i}\left(r_{i+1}+\ldots+r_{l-1}\right)=r_{i}+r_{i+1}+\ldots+r_{l-1}$ for $i=1 \ldots l-2$ we also have

$$
w_{1} w_{2} \ldots w_{l-2}\left(r_{l-1}\right)=w_{1} w_{2} \ldots w_{l-3}\left(r_{l-2}+r_{l-1}\right)=r_{1}+\ldots+r_{l-2}+r_{l-1} .
$$

This gives $w\left(r_{l-1}\right)=r_{1}+r_{2}+\ldots+r_{l}$. 
Using all these observations we can determine a part of the orbit of $\langle w\rangle$ containing $r_{1}$, which is the following:

$$
\rightarrow r_{1} \stackrel{\mathrm{w}}{\longrightarrow} r_{2} \stackrel{\mathrm{w}}{\longrightarrow} \cdots \stackrel{\mathrm{w}}{\longrightarrow} r_{l-1} \stackrel{\mathrm{w}}{\longrightarrow} r_{l}+r_{l-1}+r_{l-2}+\ldots+r_{1} \rightarrow
$$

Lemma 9. $x_{1}(1), n_{w}$ and $h_{r_{1}}(\lambda)$ generate the Chevalley group $G$.

Proof. The proof can be found in [13].

The construction is almost the same as in the case $A_{l}$. Let

$$
\Gamma_{c}=\operatorname{Cay}\left(G,\left\{x_{1}(1), x_{1}(-1), n_{w}, n_{w}^{-1}, h_{r_{1}}(\lambda), h_{r_{1}}(\lambda)^{-1}\right\}\right) .
$$

Let

$$
K_{c}=\left\langle X_{r_{2}}, X_{-r_{2}}, X_{r_{3}}, X_{-r_{3}}, \ldots, X_{r_{l}}, X_{-r_{l}}\right\rangle
$$

and let

$$
S_{c}=\cup_{i=0}^{l-2} K_{c} n_{w}^{i}
$$

Lemma 10. $\frac{\left|\partial\left(S_{c}\right)\right|}{\left|S_{c}\right|} \leq \frac{6}{l-1}$

Proof. Similarly to the previous cases $n_{w}^{-i} K_{c} n_{w}^{i}$ contains $n_{w}^{-i} X_{r_{i+1}} n_{w}^{i}=X_{r_{1}}$ for $1 \leq i \leq l-2$ which gives that $n_{w}^{i}$ is not in $K_{c}$ if $1 \leq i \leq l-2$. This proves that $\left|S_{c}\right|=(l-1)\left|K_{c}\right|$.

Again, $K_{c} n_{w}^{i} n_{w} \subset S_{c}$ if $1 \leq i \leq l-3$ and $K_{c} n_{w}^{i} n_{w}^{-1} \subset S_{c}$ if $i \neq 0$.

It is also easy to verify that $n_{w}^{i} x_{1}(1)^{ \pm 1} n_{w}^{-i}=\left(x_{i+1}(t)\right)^{ \pm 1}$ for some $t \in$ $G F(q)^{*}$. Therefore $n_{w}^{i} x_{1}(1)^{ \pm 1} n_{w}^{-i} \in X_{r_{i+1}}$ and $n_{w}^{i} h_{r_{1}}(\lambda)^{ \pm 1} n_{w}^{-i}$ are in the subgroup generated by $X_{r_{i+1}}$ and $X_{-r_{i+1}}$ for $i=1, \ldots, l-2$. Thus the elements of the right cosets $K_{c} n_{w}^{i} x_{1}(1)^{ \pm 1}$ and $K_{c} n_{w}^{i} h_{r_{1}}(\lambda)^{ \pm 1}$ are in $S_{c}$ if $1 \leq i \leq l-2$. This proves that $\partial S_{c} \subseteq K_{c} n_{w}^{l-1} \cup K_{c} n_{w}^{-1} \cup K_{c} x_{1}(1) \cup K_{c} x_{1}(1)^{-1} \cup K_{c} h_{r_{1}}(\lambda) \cup$ $K_{c} h_{r_{1}}(\lambda)^{-1}$, which is the union of 6 right cosets of $K_{c}$. Thus $\left|\partial S_{c}\right| \leq 6\left|K_{c}\right|$.

\section{$3.4 D_{l}$}

The Dynkin diagram in this case is the following:

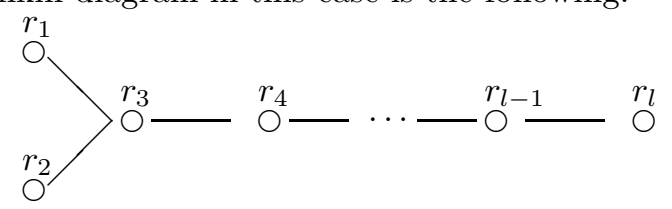

Lemma 11. (a) $x_{r_{1}}(1), n_{w}$ and $h_{r_{1}}(\lambda)$ generate the Chevalley group $G$ if the rank of $G$ is odd.

(b) $x_{-r_{1}}(1), x_{r_{1}}(1), x_{3}(1), n_{w}$ and $h_{r_{1}}(\lambda)$ generate the Chevalley group $G$ if the rank of $G$ is even.

Proof. The proof can be found in [13]. 
First, we describe a part of the orbit of $\langle w\rangle$ which contains $r_{1}$. The root $r_{1}$ is orthogonal to $r_{4}, \ldots, r_{l}$ hence $w\left(r_{1}\right)=w_{1} w_{2} w_{3}\left(r_{1}\right)$ so we have

$w_{1} w_{2} w_{3}\left(r_{1}\right)=w_{1} w_{2}\left(r_{1}+r_{3}\right)=w_{1}\left(r_{1}+r_{2}+r_{3}\right)=-r_{1}+r_{2}+r_{3}+r_{1}=r_{2}+r_{3}$

and similarly

$$
w\left(r_{2}\right)=w_{1} w_{2} w_{3}\left(r_{2}\right)=w_{1} w_{2}\left(r_{2}+r_{3}\right)=w_{1}\left(-r_{2}+r_{3}+r_{2}\right)=r_{3}+r_{1} .
$$

Using Lemma 1 we get $w\left(r_{i}\right)=r_{i+1}$ for $i=3, \ldots, l-1$. This gives that both $w^{i}\left(r_{1}\right)$ and $w^{i}\left(r_{2}\right)$ are of the form

$$
r_{i+2}+r_{i+1}+\ldots+r_{3}+y
$$

where $y=r_{1}$ or $y=r_{2}$.

\subsubsection{Odd case}

Let us assume that $l$ is odd.

Let

$$
\Gamma_{d}=\operatorname{Cay}\left(G,\left\{x_{r_{1}}(1), x_{r_{1}}(1)^{-1}, n_{w}, n_{w}^{-1}, h_{r_{1}}(\lambda), h_{r_{1}}(\lambda)^{-1}\right\}\right) .
$$

Let

$$
K_{d}=\left\langle X_{r_{1}}, X_{-r_{1}}, X_{r_{2}}, X_{-r_{2}}, \ldots, X_{r_{l-1}}, X_{-r_{l-1}}\right\rangle
$$

and let

$$
S_{d}=\cup_{i=0}^{l-3} K_{d} n_{w}^{i}
$$

Lemma 12. $\frac{\left|\partial\left(S_{d}\right)\right|}{\left|S_{d}\right|} \leq \frac{2}{l-2}$

Proof. It is easy to see that if $0 \leq i \leq l-3$, then $n_{w}^{i} x_{r_{1}}(1)^{ \pm 1} n_{w}^{-i}=x_{w^{i}\left(r_{1}\right)}(t)^{ \pm 1} \in$ $K_{d}$ for some $t \in G F(q)^{*}$ since by (8) the root $w^{i}\left(r_{1}\right)$ is a linear combination with integer coefficients of the fundamental roots $r_{1}, r_{2}, \ldots, r_{l-1}$ and similarly $n_{w}^{i} h_{r_{1}}(\lambda)^{ \pm 1} n_{w}^{-i} \in K_{d}$. It follows that $\partial S_{d} \subseteq K_{d} n_{w}^{l-2} \cup K_{d} n_{w}^{-1}$.

It remains to show that for $S_{d}$ is the union of $l-3$ pairwise disjoint cosets of $K_{d}$. Again, $n_{w}^{i} K_{d} n_{w}^{-i}$ contains the subgroup $n_{w}^{i} X_{r_{l-i}} n_{w}^{-i}=X_{r_{l}}$ if $1 \leq i \leq l-3$ which shows that $n_{w}^{i} \notin K_{d}$.

\subsubsection{Even case}

Let us assume that $l$ is even.

Let

$$
\Gamma_{d}^{\prime}=\operatorname{Cay}\left(G,\left\{x_{-r_{1}}( \pm 1), x_{-r_{1}}( \pm 1), x_{r_{3}}( \pm 1), n_{w}, n_{w}^{-1}, h_{r_{1}}(\lambda), h_{r_{1}}(\lambda)^{-1}\right\}\right) .
$$

Let

$$
K_{d}^{\prime}=\left\langle X_{r_{1}}, X_{-r_{1}}, X_{r_{2}}, X_{-r_{2}}, \ldots, X_{r_{l-1}}, X_{-r_{l-1}}\right\rangle
$$

and let

$$
S_{d}^{\prime}=\cup_{i=0}^{l-4} K_{d}^{\prime} n_{w}^{i} .
$$


Lemma 13. $\frac{\left|\partial\left(S_{d}^{\prime}\right)\right|}{\left|S_{d}^{\prime}\right|} \leq \frac{2}{l-3}$

Proof. It is clear that $w^{i}\left(-r_{1}\right)=-w^{i}\left(r_{1}\right)$ and hence $w^{i}\left(-r_{1}\right)$ is in the root system generated by the roots $r_{1}, r_{2}, \ldots, r_{l-1}$ if $1 \leq i \leq l-4$. This shows that $n_{w}^{i} x_{ \pm r_{1}}( \pm 1) n_{w}^{-i}=x_{w^{i}\left( \pm r_{1}\right)}(t) \in K_{d}^{\prime}$ for some $t \in G F(q)^{*}$.

It was proved in Lemma 12 that $n_{w}^{i} h_{r_{1}}(\lambda)^{ \pm 1} n_{w}^{-i} \in K_{d}^{\prime}$ if $0 \leq i \leq l-4$. Finally, by Lemma $1 n_{w}^{i} x_{r_{3}}( \pm 1) n_{w}^{-i}=x_{r_{3+i}}(t)$ for some $t \in K^{*}$ which is in $K_{d}^{\prime}$ if $0 \leq i \leq l-4$. It follows that $\partial S_{d}^{\prime} \subseteq K_{d}^{\prime} n_{w}^{l-3} \cup K_{d}^{\prime} n_{w}^{-1}$.

It remains to show that $S_{d}^{\prime}$ is the union of $l-3$ pairwise disjoint cosets of $K_{d}^{\prime}$. This is clear since if $1 \leq i \leq l-4$, then $n_{w}^{i} K_{d}^{\prime} n_{w}^{-i}$ contains the subgroup $X_{r_{l}}$ which shows that $n_{w}^{i} \notin K_{d}^{\prime}$.

\section{Twisted groups}

The twisted groups can be obtained as subgroups of Chevalley groups. In order to define twisted groups we need to find a non-trivial symmetry $\rho$ of the Dynkin diagram. We restrict our attention to those twisted groups which are defined using a symmetry of order 2 and we also assume that the roots in $\Phi$ have the same length. It is well known that such an symmetry $\rho$ can be extended to a unique isometry $\tau$ of $V$ which is the vector space spanned by $\Phi$. We assume that $\operatorname{Aut}\left(G F(q)^{*}\right)$ contains an element of order 2. Then the Chevalley group $G$ has an automorphism of order 2 , which we denote by $\alpha$ such that $x_{r}(t)^{\alpha}=x_{\bar{r}}(\bar{k})$ for every $r \in \pm \Pi$ and $k \in K$, where $\bar{k}=\tau(k)$ and $\bar{r}=\rho(r)$.

The subgroup $U^{1}$ is the set of elements $u \in U$ such that $u^{\alpha}=u$ and similarly $V^{1}=\left\{v \in V \mid v^{\alpha}=v\right\}$. The twisted group $G^{1}$ is generated by $U^{1}$ and $V^{1}$. The subgroups $H^{1}$ and $N^{1}$ are defined as the intersection of $G^{1}$ with $H$ and $N$, respectively. We denote by $W^{1}$ the elements $w$ of the Weyl group $W$ such that $\tau w \tau^{-1}=w$. There is a natural isomorphism of the group $W^{1}$ to $N^{1} / H^{1}$ and we denote by $n_{w}^{1}$ the element of $N^{1} \leq N$ which corresponds to $w^{1} \in W^{1}$.

The set of positive roots $\Phi^{+}$has a partition where the elements of the partition are of the following form:

$$
\begin{aligned}
& Z=\left\{r \mid r \in \Phi^{+}\right\} \text {if } \bar{r}=r \\
& Z=\left\{r, \bar{r} \mid r \in \Phi^{+} \text {and } r+\bar{r} \notin \Phi\right\} \\
& Z=\left\{r, \bar{r}, r+\bar{r} \mid r \in \Phi^{+} \text {and } r+\bar{r} \in \Phi\right\} .
\end{aligned}
$$

We denote by $\Pi^{1}$ the collection of sets which are elements of the partition. For each set $Z$ in the partition there is a unique element $w_{Z} \in W^{1}$ which is generated by $\left\{w_{r} \mid r \in Z\right\}$ such that $w(Z)=-Z$. These elements are the following:

$$
\begin{aligned}
& w_{Z}=w_{r} \text { if } Z=\left\{r \mid r \in \Phi^{+}\right\} \\
& w_{Z}=w_{r} w_{\bar{r}} \text { if } Z=\left\{r, \bar{r} \mid r \in \Phi^{+} \text {and } r+\bar{r} \notin \Phi\right\} \\
& w_{Z}=w_{r+\bar{r}}=w_{r} w_{\bar{r}} w_{r} \text { if } Z=\left\{r, \bar{r}, r+\bar{r} \mid r \in \Phi^{+} \text {and } r+\bar{r} \in \Phi\right\} .
\end{aligned}
$$


Every element of $\Pi^{1}$ can be obtained as $w(Z)$, where $w \in W^{1}$ and $Z$ contains a fundamental root. Those sets which contain a fundamental root are called fundamental sets. Moreover, $W^{1}$ is generated by $\left\{w_{Z} \mid Z \in \Pi^{1}\right\}$.

For every $Z \in \Pi^{1}$ we denote by $X_{Z}$ the subgroup generated by the root subgroups $X_{r}$ for $r \in Z$ and $X_{Z}^{1}=X_{Z} \cap G^{1}$.

\section{1 $A_{2 n-1}^{1}$}

The fundamental sets in this case are the following:

$$
Z_{n}=\left\{r_{n}\right\}, Z_{i}=\left\{r_{i}, r_{2 n-i}\right\} \text { for } 1 \leq i \leq n-1,
$$

and the corresponding elements of the Weyl group $W^{1}$ are:

$$
w_{Z_{n}}=w_{n}, \text { and } w_{Z_{i}}=w_{i} w_{2 n-i} \text { for } 1 \leq i \leq n-1 .
$$

We may assume (see [2, p.233.]) that the subgroups defined above are of the following form :

$$
\begin{aligned}
& X_{Z}^{1}=\left\{x_{r}(t) \mid t=\bar{t}\right\} \text { if } Z=\{r\} \\
& X_{Z}^{1}=\left\{x_{r}(t) x_{\bar{r}}(\bar{t}) \mid t \in K\right\} \text { if } Z=\{r, \bar{r}\} .
\end{aligned}
$$

Let $n_{w}^{1}=n_{w_{1}^{1}} n_{w_{2}^{1}} \ldots$ and $h_{e}=h_{r_{1}}(t) h_{\overline{r_{1}}}(\bar{t})$, where $t$ generates $K^{*}$. In the following in order to simplify notation we write $n_{w}$ instead of $n_{w}^{1}$.

We also define $x_{e}=x_{r_{1}}(1) x_{r_{2 n-1}}(1)$ which is an element of $X_{Z_{1}}^{1}$ and which can also be written as $x_{r_{1}}(1) x_{r_{1}}(1)^{\alpha}=x_{r_{1}}(1) x_{\overline{r_{1}}}(1)$.

Lemma 14. $x_{e}, n_{w}$ and $h_{e}$ generate the group $G^{1}$.

Proof. The proof can be found in [13.

Let

$$
\Gamma_{e}=\operatorname{Cay}\left(G,\left\{x_{e}, x_{e}^{-1}, n_{w}, n_{w}^{-1}, h_{e}, h_{e}^{-1}\right\}\right) .
$$

Let

$$
K_{e}=\left\langle X_{Z_{2}}^{1}, X_{-Z_{2}}^{1}, X_{Z_{3}}^{1}, X_{-Z_{3}}^{1}, \ldots, X_{Z_{n}}^{1}, X_{-Z_{n}}^{1}\right\rangle
$$

and let

$$
S_{e}=\cup_{i=0}^{n-2} K_{e} n_{w}^{i} .
$$

$K_{e}$ can be considered as a twisted group which is a subgroup of the Chevalley group generated by the root subgroups $X_{r_{2}}, X_{-r_{2}}, \ldots, X_{r_{2 n-2}}, X_{-r_{2 n-2}}$. The corresponding set of fundamental roots is $\rho$-invariant and we denote by $\Phi_{2 n-3}$ the root system generated by these roots. The restriction of $\rho$ to the set $\left\{r_{2}, r_{3}, \ldots, r_{2 n-2}\right\}$ gives a symmetry of the Dynkin diagram of these roots which extends to an isometry. This isometry is the restriction of $\tau$. This gives that for $Z \in \Pi^{1}$ the subgroup $X_{Z}^{1}$ is a subgroup of $K_{e}$ if and only if $Z \subset \Phi_{2 n-3}$. Clearly, $h_{r}(t)$ is in $\left\langle X_{Z}^{1}, X_{-Z}^{1}\right\rangle \subset G^{1}$ if $Z=\{r\}$ with $r=\bar{r}$ and if $Z=\{r, \bar{r}\}$, then there is homomorphism of $S L_{2}(K)$ onto $\left\langle X_{Z}^{1}, X_{-Z}^{1}\right\rangle \subset G^{1}$ which shows that $x_{r}(t) x_{\bar{r}}(\bar{t}) \in G^{1}$ and $h_{r}(t) h_{\bar{r}}(\bar{t}) \in G^{1}$. 
Conjugating by $n_{w}^{i} \in N^{1}$ we get the following:

$$
\begin{aligned}
n_{w}^{-i} X_{Z}^{1} n_{w}^{i} & =n_{w}^{-i}\left(X_{Z} \cap G^{1}\right) n_{w}^{i}=n_{w}^{-i} X_{Z} n_{w}^{i} \cap n_{w}^{-i} G^{1} n_{w}^{i}=X_{w^{-i}(Z)} \cap G^{1} \\
& =X_{w^{-i}(Z)}^{1} .
\end{aligned}
$$

Lemma 15. $\frac{\left|\partial\left(S_{e}\right)\right|}{\left|S_{e}\right|} \leq \frac{6}{n-1}$

Proof. We claim that $S_{e}$ is the union of $n-1$ disjoint subsets. $K_{e} n_{w}^{j}=K_{e} n_{w}^{j^{\prime}}$ if and only if $n_{w}^{j-j^{\prime}} \in K_{e}$ so we have to show that $n_{w}^{i} \notin K_{e}$ if $1 \leq i \leq n-2$. We claim that $w^{i}\left(r_{1}\right)=r_{i+1}$ if $1 \leq i \leq n-2$. If $k \leq n-3$, then

$$
w\left(r_{k}\right)=w_{1} w_{2 n-1} \ldots w_{k} w_{2 n-k} w_{k+1}\left(r_{k}\right)
$$

since $r_{k}$ is orthogonal to $r_{j}$ if $j \geq k+2$. Therefore

$$
\begin{aligned}
w\left(r_{k}\right) & =w_{1} w_{2 n-1} \ldots w_{k}\left(r_{k}+r_{k+1}\right) \\
& =w_{1} w_{2 n-1} \ldots w_{k-1}\left(r_{k+1}\right)=r_{k+1}
\end{aligned}
$$

since $r_{k+1}$ is orthogonal to the roots $r_{2 n-k}, \ldots, r_{2 n-1}$ and $r_{k+1}$ is orthogonal to $r_{1}, \ldots, r_{k-1}$. It follows that $w^{-i}\left(Z_{i+1}\right)=Z_{1}$ and hence by equation (9) $X_{Z_{1}}^{1} \subset n_{w}{ }^{-i} S_{e} n_{w}^{i}$ if $1 \leq i \leq n-2$. This proves that $n_{w}^{i} \notin K_{e}$ if $1 \leq i \leq n-2$ hence $\left|S_{e}\right|=(n-1)\left|K_{e}\right|$.

It is easy to see that $S_{e}$ contains $K_{e} n_{w}^{i} n_{w}$ if $i=0,1, \ldots, n-3$ and $S_{e}$ contains $K_{e} n_{w}^{i} n_{w}^{-1}$ if $i=1,2, \ldots, n-2$.

We use again the fact that $K_{e} n_{w}^{i} g=K_{e} n_{w}^{i}$ if and only if $n_{w}^{i} g n_{w}^{-i} \in K_{e}$. Since $n_{w}^{i} x_{r_{1}}(1) n_{w}^{-i}=x_{w\left(r_{1}\right)}(\lambda)$ for some $\lambda \in K$ and $x_{e}=x_{r_{1}}(1) x_{r_{1}}(1)^{\alpha}$ we have

$$
\begin{aligned}
& n_{w}^{i} x_{r_{1}}(1) x_{r_{1}}(1)^{\alpha} n_{w}^{-i}=n_{w}^{i} x_{r_{1}}(1) n_{w}^{-i} n_{w}^{i} x_{r_{1}}(1)^{\alpha} n_{w}^{-i} \\
= & n_{w}^{i} x_{r_{1}}(1) n_{w}^{-i}\left(n_{w}^{i} x_{r_{1}}(1) n_{w}^{-i}\right)^{\alpha}=x_{w^{i}\left(r_{1}\right)} x_{w^{i}\left(r_{1}\right)}(\lambda)^{\alpha}=x_{r_{i+1}} x_{r_{i+1}}(\lambda)^{\alpha} .
\end{aligned}
$$

This shows that $n_{w}^{i} x_{e} n_{w}^{-i} \in X_{Z_{i+1}}^{1}$ which proves that if $i=1,2, \ldots, n-2$, then $n_{w}^{i} x_{e}^{ \pm 1} n_{w}^{-i} \in K_{e}$ and hence $K_{e} n_{w}^{i} x_{e}^{ \pm 1}=K_{e} n_{w}^{i}$ since $Z_{i+1} \subset \Phi_{2 n-3}$.

We also have $n_{w}^{i} h_{r_{1}}(t) h_{\overline{r_{1}}}(\bar{t}) n_{w}^{-i}=h_{r_{i+1}}(\theta) h_{w^{i}\left(\overline{r_{1}}\right)}\left(\theta^{\prime}\right)$ for some $\theta, \theta^{\prime} \in K$. Using the fact that $w \in W^{1}$ we have $w^{i}\left(\overline{r_{1}}\right)=\overline{w^{i}\left(r_{1}\right)}$ so $h_{r_{i+1}}(\theta) h_{w^{i}\left(\overline{r_{1}}\right)}\left(\theta^{\prime}\right)=$ $h_{r_{i+1}}(\theta) h_{\overline{r_{i+1}}}\left(\theta^{\prime}\right)$. Clearly, $n_{w}^{i} h_{e} n_{w}^{-i} \in H^{1}$. Thus $\theta^{\prime}=\bar{\theta}$ and $n_{w}^{i} h_{e}^{ \pm 1} n_{w}^{-i}=$ $\left(h_{r_{i+1}}(\theta) h \frac{}{\left(r_{i+1}\right)}(\overline{\theta)})^{ \pm 1} \in K_{e}\right.$ since $r_{i+1} \in \Phi_{2 n-3}$ if $i=1, \ldots, n-2$. This proves that $K_{e} n_{w}^{i} h_{e}^{ \pm 1}=K_{e} n_{w}^{i}$ if $i=1, \ldots, n-2$ and hence $\partial S_{e} \subset K_{e} n_{w}^{n-1} \cup K_{e} n_{w}^{-1} \cup$ $K_{e} x_{e} \cup K_{e} x_{e}^{-1} \cup K_{e} h_{e} \cup K_{e} h_{e}^{-1}$, finishing the proof of Lemma 15.

\section{$4.2 \quad D_{n}^{1}$}

The fundamental sets in this case are the following:

$$
Z_{1}=\left\{r_{1}, r_{2}\right\}, Z_{i}=\left\{r_{i+1}\right\} \text { for } 2 \leq i \leq n-1,
$$


and the corresponding elements of the Weyl group $W^{1}$ are:

$$
w_{Z_{1}}=w_{1} w_{2} \text {, and } w_{Z_{i}}=w_{i+1} \text { for } 2 \leq i \leq n-1 .
$$

Let $n_{w}=n_{w_{1}^{1}} n_{w_{2}^{1}} \ldots n_{w_{n-1}^{1}}$ and $h_{f}=h_{r_{1}}(t) h_{\overline{r_{1}}}(\bar{t})$, where $t$ generates $K^{*}$.

We also define $x_{f}=x_{r_{1}}(1) x_{r_{2}}(1)$ which can also written as $x_{r_{1}}(1) x_{r_{1}}(1)^{\alpha}=$ $x_{r_{1}}(1) x_{\overline{r_{1}}}(1)$.

Lemma 16. $x_{f}, n_{w}$ and $h_{f}$ generate the group $G^{1}$.

Proof. The proof can be found in [13].

Let

$$
\Gamma_{f}=\operatorname{Cay}\left(G,\left\{x_{f}, x_{f}^{-1}, n_{w}, n_{w}^{-1}, h_{f}, h_{f}^{-1}\right\}\right) .
$$

Let

$$
K_{f}=\left\langle X_{Z_{1}}^{1}, X_{-Z_{1}}^{1}, X_{Z_{2}}^{1}, X_{-Z_{2}}^{1}, \ldots, X_{Z_{n-2}}^{1}, X_{-Z_{n-2}}^{1}\right\rangle
$$

and let

$$
S_{f}=\cup_{i=0}^{n-3} K_{f} n_{w}^{i} .
$$

We denote by $\Phi_{n-1}$ the root system generated by the fundamental roots $r_{1}, r_{2}, \ldots, r_{n-1}$.

Lemma 17. $\frac{\left|\partial\left(S_{f}\right)\right|}{\left|S_{f}\right|} \leq \frac{2}{n-2}$

Proof. The Coxeter element in this case is exactly the same as in subsection 3.4. This gives that $n_{w}^{i}\left(r_{n-i}\right)=r_{n}$ for $0 \leq i \leq n-3$. The fundamental sets $Z_{2}, Z_{3}, \ldots, Z_{n-1}$ consist of only one element thus $n_{w}^{i} S_{f} n_{w}^{-i}$ contains $X_{w^{i}\left(Z_{n-1-i}\right)}^{1}=X_{w^{i}\left(r_{n-i}\right)}=X_{r_{n}}=X_{Z_{n-1}}^{1}$ if $1 \leq i \leq n-3$ since $S_{f}$ contains $X_{n-i}^{1}$. This proves that if $1 \leq i \leq n-3$, then $n_{w}^{i} \notin K_{f}$. Thus $S_{f}$ is the union of $n-2$ disjoint subsets of the same cardinality. Therefore $\left|S_{f}\right|=(n-2)\left|K_{f}\right|$.

Using the definiton of $S_{f}$ one can see that $K_{f} n_{w}^{i} n_{w} \subset S_{f}$ if $i=0,1, \ldots, n-4$ and $K_{f} n_{w}^{i} n_{w}^{-1} \subset S_{f}$ if $i=1, \ldots, n-3$.

The elements $n_{w}^{i} x_{f} n_{w}^{-i}$ are of the form $x_{r}(t) x_{\bar{r}}( \pm \bar{t})$ for some $r \in \Phi$ and $t \in K^{*}$. In order to prove that these elements are in $K_{f}$ for $i=0,1, \ldots, n-3$ we only have to show that $r \in \Phi_{n-1}$. Using the fact that the Coxeter element in this case is the same as in Section 3.4 we have that both $w^{i}\left(r_{1}\right)$ and $w^{i}\left(r_{2}\right)$ are of the form $r_{1}+r_{3}+r_{4}+\ldots+r_{i+1}$ or $r_{2}+r_{3}+r_{4}+\ldots+r_{i+1}$. These roots are clearly in the root system generated by the fundamental roots $r_{1}, r_{2}, \ldots, r_{l-1}$ if $i \leq n-2$. This proves that $n_{w}^{i} x_{f}^{ \pm} n_{w}^{-i}$ is in $K_{f}$ if $0 \leq i \leq n-3$ and hence $S_{f} x_{f}^{ \pm} \subset S_{f}$.

Similarly, the elements $n_{w}^{i} h_{f} n_{w}^{-i}$ are of the form $h_{r}(t) h_{\bar{r}}(\bar{t})$ for some $r \in \Phi$ and $t \in K^{*}$ and it is easy to see that $r \in \Phi_{n-1}$ if $0 \leq i \leq n-3$. This proves that $n_{w}^{i} h_{f}^{ \pm} n_{w}^{-i}$ is in $K_{f}$ if $0 \leq i \leq n-3$ and hence $S_{f} h_{f}^{ \pm} \subset S_{f}$. 


\section{$4.3 \quad A_{2 n}^{1}$}

The fundamental sets are the following:

$$
Z_{1}=\left\{r_{n}, r_{n+1}, r_{n}+r_{n+1}\right\}, Z_{i}=\left\{r_{n+1-i}, r_{n+i}\right\} \text { for } 2 \leq i \leq n .
$$

Let $n_{w}^{1}=n_{w_{1}^{1}} n_{w_{2}^{1}} \ldots n_{w_{n}^{1}}$ and $h_{g}=h_{r_{n}}(t) h_{\overline{r_{n}}}(\bar{t})$, where $t$ generates $K^{*}$. We also define $x_{g}=x_{r_{n}}(1) x_{r_{n+1}}(1) x_{r_{n}+r_{n+1}}(k)$ with $k+\bar{k}=1$.

Lemma 18. $x_{g}, n_{w}$ and $h_{g}$ generate the group $G^{1}$.

Proof. The proof can be found in [13].

Let

$$
\Gamma_{g}=\operatorname{Cay}\left(G,\left\{x_{g}, x_{g}^{-1}, n_{w}, n_{w}^{-1}, h_{g}, h_{g}^{-1}\right\}\right) .
$$

Let

$$
K_{g}=\left\langle X_{Z_{1}}^{1}, X_{-Z_{1}}^{1}, X_{Z_{2}}^{1}, X_{-Z_{2}}^{1}, \ldots, X_{Z_{n-1}}^{1}, X_{-Z_{n-1}}^{1}\right\rangle
$$

and let

$$
S_{g}=\cup_{i=0}^{n-2} K_{g} n_{w}^{i}
$$

Lemma 19. $\frac{\left|\partial\left(S_{g}\right)\right|}{\left|S_{g}\right|} \leq \frac{2}{n-1}$

Proof. First, we show that $S_{g}$ is the union of $n-1$ disjoint subsets of the same cardinality. It is enough to show that $n_{w}^{i} \notin K_{g}$ for $i=1, \ldots, n-2$. This will be done by proving that $X_{Z_{n}}^{1}$ is contained in $n_{w}^{i} K_{g} n_{w}^{-i}$. Using equation (9) we only have to show that $w^{i}\left(Z_{n-i}\right)=Z_{n}$ for $i=1, \ldots n-2$.

The fundamental root $r_{k+1}$ is contained in $Z_{n-k}$. Let us assume that $1 \leq$ $k \leq n-2$.

$$
\begin{aligned}
w\left(r_{k+1}\right) & =w_{n} w_{n+1} w_{n} w_{n-1} w_{n+2} \ldots w_{1} w_{2 n}\left(r_{k+1}\right) \\
& =w_{n} w_{n+1} w_{n} w_{n-1} w_{n+2} \ldots w_{k+1} w_{2 n-k} w_{k}\left(r_{k+1}\right)
\end{aligned}
$$

since $r_{k+1}$ is orthogonal to the roots $r_{j}$ if $j>n$ or $j<k-1$. Clearly, $w_{k+1} w_{2 n-k} w_{k}\left(r_{k+1}\right)=r_{k}$ so

$$
w\left(r_{k+1}\right)=w_{n} w_{n+1} w_{n} \ldots w_{k+2} w_{2 n-k-1}\left(r_{k}\right)=r_{k}
$$

since the remaining reflections fix $r_{k}$.

One can see by induction that $w^{i}\left(r_{i+1}\right)=r_{1}$ for $i=1, \ldots n-2$ and since $w \in$ $W^{1}$ we have $w^{i}\left(\overline{r_{i+1}}\right)=\overline{w^{i}\left(r_{i+1}\right)}=r_{2 n}$ and hence $w^{i}\left(Z_{n-i}\right)=Z_{n}$. This proves that for $i=1, \ldots, n-2$ the subgroup $n_{w}^{i}\left(K_{g}\right) n_{w}^{-i}$ contains $X_{Z_{n}}^{1}$. Therefore $\left|S_{g}\right|=(n-1)\left|K_{g}\right|$.

The definition of $S_{g}$ shows that $K_{g} n_{w}^{i} n_{w} \subset S_{g}$ if $i \neq n-2$ and $K_{g} n_{w}^{i} n_{w}^{-1} \subset S_{g}$ if $i \neq 0$. It remains to investigate the elements of the form $n_{w}^{i} x_{g}^{ \pm} n_{w}^{-i}$ and $n_{w}^{i} h_{g}^{ \pm} n_{w}^{-i}$. 
We claim, that $w^{i}\left(r_{n}\right)=r_{n}+r_{n-1}+\ldots+r_{n-i}$ if $i \leq n-2$. Using the orthogonality of the fundamental vectors $r_{j}, r_{k}$, where $|j-k| \geq 2$ we get the following:

$$
\begin{aligned}
w\left(r_{n}\right) & =w_{n} w_{n+1} w_{n} w_{n-1} w_{n+2} \ldots w_{1} w_{2 n}\left(r_{n}\right) \\
& =w_{n} w_{n+1} w_{n} w_{n-1}\left(r_{n}\right)=w_{n} w_{n+1}\left(r_{n-1}\right)=r_{n-1}+r_{n} .
\end{aligned}
$$

Similarly, if $1 \leq k \leq n-2$, then

$$
\begin{aligned}
w\left(r_{n-k}\right) & =w_{n} w_{n+1} w_{n} w_{n-1} w_{n+2} \ldots w_{1} w_{2 n}\left(r_{n-k}\right) \\
& =w_{n} w_{n+1} w_{n} \ldots w_{n-k} w_{n+k+1} w_{n-k-1}\left(r_{n-k}\right) \\
& =w_{n} w_{n+1} w_{n} \ldots w_{n-k+1}\left(r_{n-k-1}\right)=r_{n-k-1} .
\end{aligned}
$$

Since $w$ is linear we get using (10) and (11) that

$$
w^{i}\left(r_{n}\right)=r_{n}+r_{n-1}+\ldots+r_{n-i} .
$$

By observing equations (12) one can see that if $i=0, \ldots, n-2$, then both $r_{1}$ and $r_{2 n}$ are orthogonal to $w^{i}\left(r_{n}\right)$ and similarly $r_{1}$ and $r_{2 n}$ are orthogonal to $w^{i}\left(r_{n+1}\right)=w^{i}\left(\overline{r_{n}}\right)=\overline{w^{i}\left(r_{n}\right)}=r_{n+i+1}$. This shows that for $w^{\prime}=$ $w_{n} w_{n+1} w_{n} w_{n-1} w_{n+2} \ldots w_{2} w_{2 n-1}$ we have $w^{i}\left(r_{n}\right)=\left(w^{\prime}\right)^{i}\left(r_{n}\right)$ and $w^{i}\left(r_{n+1}\right)=$ $\left(w^{\prime}\right)^{i}\left(r_{n+1}\right)$. Therefore $w^{i}\left(r_{n}+r_{n+1}\right)=\left(w^{\prime}\right)^{i}\left(r_{n}+r_{n+1}\right)$. Moreover, $n_{w}^{i} x_{g} n_{w}^{-i}=$ $n_{w^{\prime}}^{i} x_{g} n_{w^{\prime}}^{-i}$ and $n_{w}^{i} h_{g} n_{w}^{-i}=n_{w^{\prime}}^{i} h_{g} n_{w^{\prime}}^{-i}$.

Clearly, $n_{w^{\prime}} \in K_{g}$ and hence the elements $n_{w^{\prime}}^{i} x_{g}^{ \pm} n_{w^{\prime}}^{-i}$ and $n_{w^{\prime}}^{i} h_{g}^{ \pm} n_{w^{\prime}}^{-i}$ are in $K_{g}$ if $i=0,1, \ldots n-2$.

In order to finish the proof of Theorem (1) we have to verify that the for those sets $S$ for which boundary $\partial(S)$ is relatively small we have $|S| \leq \frac{|G|}{2}$. The order of the investigated simple groups is the following:

$$
\begin{array}{rc}
A_{l}(q): & \frac{1}{(n+1, q-1)} q^{\frac{n(n-1)}{2}} \prod_{i=1}^{n}\left(q^{i+1}-1\right) \\
B_{l}(q): & \frac{1}{(2, q-1)} q^{n^{2}} \prod_{i=1}^{n}\left(q^{2 i}-1\right) \\
C_{l}(q): & \frac{1}{(2, q-1)} q^{n^{2}} \prod_{i=1}^{n}\left(q^{2 i}-1\right) \\
D_{l}(q): & \frac{1}{\left(4, q^{n}-1\right)} q^{\frac{n(n-1)}{2}} \prod_{i=1}^{n}\left(q^{2 i}-1\right) \\
A_{l}\left(q^{2}\right)^{1}: & \frac{1}{(n+1, q+1)} q^{\frac{n(n-1)}{2}} \prod_{i=1}^{n}\left(q^{i+1}-(-1)^{i+1}\right) \\
D_{l}\left(q^{2}\right)^{1}: & \frac{1}{\left(4, q^{n}+1\right)} q^{n(n-1)}\left(q^{n}+1\right) \prod_{i=1}^{n}\left(q^{2 i}-1\right)
\end{array}
$$

It is easy to see that such a simple group can not have a subgroup of index at most $2 l$, finishing the proof of Theorem 1 .

\section{Identification}

In this section we give explicit generators of the Cayley graphs that we investigated in Section 3 and 4 . We also show how to find the subsets of the vertices $S$ for which $\partial S$ is relatively small. We only handle the case of Special Linear 
Groups which can easily be transformed to the case of the Projective Special Linear Groups which is clearly the easiest one. This example includes the original idea which was extended to several different series of simple groups. In order to show the simplicity of the original construction we forget about the machinery which was built up before.

Let

$$
A_{l}=\left(\begin{array}{ccccc}
1 & 1 & & & \\
& 1 & & & \\
& & 1 & & \\
& & & \ddots & \\
& & & & 1
\end{array}\right),
$$

where $A_{l} \in G F(q)^{(l+1) \times(l+1)}$. Let

$$
B_{l}=\left(\begin{array}{ccccc}
0 & 0 & 0 & \ldots & (-1)^{l} \\
1 & 0 & & & \\
0 & 1 & 0 & & \\
0 & 0 & \ddots & \ddots & \\
0 & & \ldots & 1 & 0
\end{array}\right) .
$$

We denote by $C_{l}$ the diagonal matrix $\operatorname{diag}\left(\frac{1}{\lambda}, \lambda, 1,1, \ldots, 1\right) \in G F(q)^{(l+1) \times(l+1)}$, where $\lambda$ generates $G F(q)^{*}$.

We denote by $e_{i, j}$ the matrix with 1 in the $(i, j)$-th position and zeros everywhere else and let $T_{i, j}(\delta)=I+\delta e_{i, j}$, where $I$ denotes the identity matrix. Using this notation we can write $A_{l}=T_{1,2}(1)$.

The standard generator $x_{r_{1}}(1)$ of the Chevalley group given in Subsection 3.1 corresponds to the matrix $A_{l}$ and the Coxeter element $n_{w}$ can be identified with $B_{l}$. Finally, $C_{l}$ plays the role of $h_{r_{1}}(\lambda)$.

Clearly, $T_{i, j}(\alpha) T_{i, j}(\beta)=T_{i, j}(\alpha+\beta)$ and $\left[T_{i, j}(\alpha), T_{j, k}(\beta)\right]=T_{i, k}(\alpha \beta)$ if $i \neq k$, where $[g, h]=g^{-1} h^{-1} g h$ denotes the commutator of $g$ and $h$.

Lemma 20. For every $l \in \mathbb{N}$ the set $\left\{A_{l}, B_{l}, C_{l}\right\}$ forms a generating set of $S L(l+1, q)$.

Proof. We fix the size of the matrices and hence we can write $A=A_{l}, B=B_{l}$ and $C=C_{l}$. Let $H=\langle A, B, C\rangle$. It is enough to verify that $T_{i, j}(\delta) \in H$ for every $i \neq j$ and $\delta \in G F(q)$.

It is easy to see that $A^{C^{k}}=T_{1,2}(1)^{C^{k}}=T_{1,2}\left(\lambda^{2 k}\right)$. Using $T_{1,2}(\mu) T_{1,2}(\eta)=$ $T_{1,2}(\mu \eta)$ we get that $T_{1,2}(\delta) \in\langle A, C\rangle \leq H$ for every $\delta \in G F(q)$. For $i \neq j$ we have $B^{k} T_{i, j}(\delta) B^{-k}=T_{i+k, j+k}( \pm \delta)$, where the indices are taken modulo $l+1$ and hence $T_{i, i+1}(\delta) \in H$ for every $1 \leq i \leq l$ and for every $\delta \in G F(q)$. This implies that for every $1<l \leq l+1$ and for every $\delta \in G F(q)$

$$
\left[\ldots\left[\left[T_{1,2}(\delta), T_{2,3}(1)\right], T_{3,4}(1)\right] \ldots, T_{k-1, k}(1)\right]=T_{1, k}(\delta) \in H .
$$

Using again the fact that $B^{k} T_{1, l}(\delta) B^{-k}=T_{1+k, l+k}( \pm \delta)$ we get that $T_{i, j}(\delta) \in H$ for every $i \neq j$ and for every $\delta \in G F(q)$. 
Let

$$
S_{0}=\left\{\left(\begin{array}{cc}
D & 0 \\
0 & E
\end{array}\right) \in S L(l+1, q) \mid D \in S L(l, q), E \in S L(1, q)\right\} .
$$

For every $1 \leq i \leq l$ we define

$$
S_{i}=S_{0} B^{i}
$$

Finally, let

$$
S=\bigcup_{i=0}^{l-1} S_{i}
$$

It is easy to see that $|S|<\frac{|S L(l+1, q)|}{2}$ if $l \geq 1$.

Lemma 21. $\frac{|\partial(S)|}{|S|} \leq \frac{6}{l}$

Proof. Every element of $S$ has exactly $l$ columns with 0 in the last row, exactly 1 column with 0 in the first $l$ and 1 in the last row. The sets $S_{i}$ are pairwise disjoint since an invertible matrix can not have a column with only zero entries. Furthermore, they all have the same cardinality since $S_{0}$ is a subgroup of $S L(n, q)$ and $S_{i}$ are right cosets of $S_{0}$ in $S L(n, q)$.

It is easy to see that $S B \backslash S \subseteq S_{0} B^{l}=S_{l}$ and $S B^{-1} \backslash S \subseteq S_{0} B^{-1}$. The remaining elements of $\partial S$ are of the form $M A, M C$ and $M A^{-1}, M C^{-1}$ where $M \in S$.

Let us assume that $M \in S_{i}$. Then

$$
M=\left(\begin{array}{ccc}
D & 0 & D^{\prime} \\
0 & 1 & 0
\end{array}\right)
$$

for some $D \in G F(q)^{l, l-i}$ and $D^{\prime} \in G F(q)^{l, i}$. Multiplying a matrix $M$ by $A$ or $A^{-1}$ from the right only modifies the second column of $M$. Therefore if $M \in S_{i}$ with $i \neq l, l-1$, then it is easy to see that $M A, M A^{-1} \in S_{i}$.

Multiplying a matrix $M$ by $C$ or $C^{-1}$ from the right only modifies the first and the second columns of $M$ thus if $M \in S_{i}$ with $i \neq l, l-1$, then $M C^{ \pm 1} \in S_{i}$.

This gives that $\partial S \subseteq S_{l} \cup S_{0} B^{-1} \cup S_{l-1} A \cup S_{l-1} A^{-1} \cup S_{l-1} C \cup S_{l-1} C^{-1}$ since $S=\cup_{i=0}^{l-1} S_{i}$.

\section{Acknowledgement}

The author is grateful to László Pyber for many valuable suggestions during the research.

\section{References}

[1] E. Breuillard, B. Green, T. Tao, Suzuki groups as expanders, arXiv:1005.0782 
[2] R. W. Carter, Simple groups of Lie type, John Wiley \& Sons, New York, Reprint of the 1972 original; A Wiley-Interscience Publication.(1989)

[3] M. Kassabov, A. Lubotzky, N. Nikolov, Finite Simple Groups as Expanders, Proc. Nat. Acad. Sci. USA 103 (2006), no. 16, 6116-6119.

[4] K. Kassabov, Universal lattices and unbounded rank expanders, Invent. Math. 170, (2007) 297-326.

[5] K. Kassabov, N. Nikolov, Universal lattices and property tau, Invent. Math. $165,(2006)$ 209-224.

[6] M. Kassabov, Symmetric group and expanders, Invent. Math. 170, (2007) 325-354.

[7] M. Kassabov, R.Riley, Diameters of Cayley graphs of Chevalley groups, Eur. J. Comb. 28 (3), (2007) 791-800.

[8] A. Lubotzky, Finite simple groups of lie type as expnders, J. Eur. Math. Soc 13, (2011) 1331-1341.

[9] A. Lubotzky, Discrete groups, expanding graphs and invariant measures, with an appendix by J. D. Rogawski, Reprint of the 1994 edition. Modern Birkhauser Classic. Bikrhauser Verlag, Basel, (2010)

[10] A. Lubotzky, Expander Graphs in Pure and Applied Mathematics, Bull. Amer. Math. Soc. 49 (2012), 113-162.

[11] A. Lubotzky, A. Zuk, On Property $(\tau)$, monograph in preparation

[12] N, Nikolov, A product decomposition for the classical quasisimple groups, J. Group Theorey 10, (2007) 43-53.

[13] Robert Steinberg, Generators for simple groups, Canad. J. Math. 14 (1962), 277-283. 